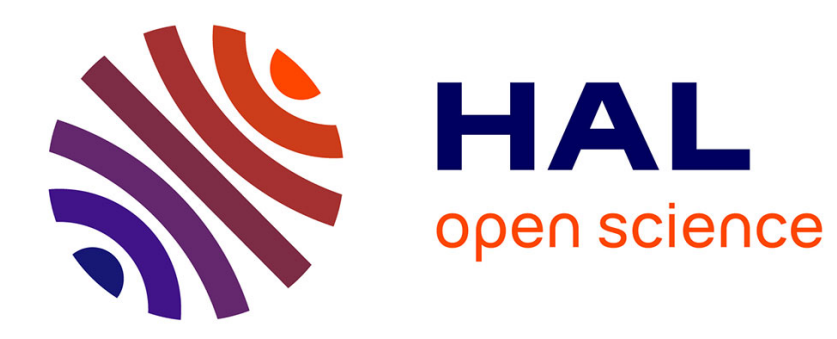

\title{
Bref aperçu historique des travaux sur la présupposition
} Sandrine Deloor

\section{To cite this version:}

Sandrine Deloor. Bref aperçu historique des travaux sur la présupposition. 2012. hal-00685769

\section{HAL Id: hal-00685769 \\ https://hal.science/hal-00685769}

Preprint submitted on 6 Apr 2012

HAL is a multi-disciplinary open access archive for the deposit and dissemination of scientific research documents, whether they are published or not. The documents may come from teaching and research institutions in France or abroad, or from public or private research centers.
L'archive ouverte pluridisciplinaire HAL, est destinée au dépôt et à la diffusion de documents scientifiques de niveau recherche, publiés ou non, émanant des établissements d'enseignement et de recherche français ou étrangers, des laboratoires publics ou privés. 
Version pre-print. A paraître dans Langages.

\title{
Bref aperçu historique des travaux sur la présupposition
}

\author{
Sandrine Deloor \\ UMR 7187 LDI, Université de Cergy-Pontoise
}

\section{Introduction}

Qu'elles apparaissent dans des manuels, des encyclopédies ou des ouvrages techniques, les synthèses sur la présupposition commencent invariablement par une liste d'exemples paradigmatiques de constructions présuppositionnelles et une liste de propriétés définitoires :

Exemples paradigmatiques de constructions présuppositionnelles :

- les descriptions définies : (1) Le roi de France est chauve présuppose < Il y a un roi de France et un seul >

- les verbes factifs : (2) Il sait que Pierre est là présuppose < Pierre est là >

- les clivées : (3) C'est Marie qui a frappé présuppose < Quelqu'un a frappé >

- les particules focales : (4) Seul Jean a accepté présuppose < Jean a accepté >

- les verbes et les adverbes marquant une succession d'états : (5) Alfred continue à fumer et (6) Alfred fume encore présupposent < Alfred fumait auparavant >

Propriétés définitoires des présupposés : Contrairement aux implications, les présupposés se conservent sous la négation, sous les opérateurs modaux, dans les conditionnelles et dans les actes de langage non assertifs.

Puis elles retracent l'histoire de la notion, en mettant en évidence les difficultés rencontrées par les différents auteurs et les solutions apportées par leurs successeurs. On a l'impression que le phénomène présuppositionnel existe en soi et que plusieurs théories successives se sont penchées dessus, résolvant un à un les problèmes identifiés.

L'approche que nous adoptons dans le présent article s'oppose à cette vision «cumulative ». Nous partons du principe qu'un concept scientifique n'existe qu'à travers la définition qu'on en donne et que des définitions différentes fondent des concepts différents. Les «problèmes à résoudre » sont eux-mêmes difficilement transposables d'une théorie à une autre car ils sont éminemment dépendants des questionnements spécifiques qui les sous-tendent. S'inscrivant dans des cadres théoriques différents, répondant à des questions différentes, les quatre approches de la présupposition que nous distinguons dans cette étude ne se sont pas substituées les unes aux autres au cours du temps : elles coexistent encore aujourd'hui et c'est pourquoi nous leur accorderons la même importance. Notre objectif sera de montrer, dans chaque cas, l'interdépendance entre les problèmes abordés et la perspective théorique adoptée : les questions que l'on se pose sont déterminées en grande partie par la théorie que l'on défend et un problème central dans un cadre devient parfois secondaire dans un autre. ${ }^{1}$

\section{Négation et échec référentiel : la controverse logico-philosophique}

La notion de présupposition a fait l'objet d'une controverse célèbre entre Frege (1892),

\footnotetext{
${ }^{1}$ Certaines des études que nous citons ont eu peu d'influence dans l'histoire de la notion (par exemple, l'approche de Fillmore 1970 exposée en 2.1) ou sont peu connues (par exemple, l'article de Karttunen \& Peters 1977 présenté en 3.1.). Il s'agit là d'une conséquence de la problématique que nous avons voulu développer : nous avons choisi les auteurs pour leur représentativité et non parce qu'ils ont trouvé leur place dans une quelconque histoire «officielle ». Dans la mesure où notre objectif était de proposer une mise en perspective des principales options théoriques possibles (et également, faute de place), le caractère plus ou moins récent des études n'a pas non plus été un critère de sélection. Pour une synthèse plus détaillée (mais aussi plus orientée d'un point de vue théorique), voir Beaver \& Geurts (2011).
} 
Version pre-print. A paraître dans Langages.

Russell (1905) et Strawson (1950). Pour les philosophes du langage en général, la signification d'un énoncé déclaratif s'identifie avec ses conditions de vérité, c'est-à-dire la spécification des circonstances dans lesquelles l'énoncé est vrai. Dans ce cadre, la présupposition d'existence véhiculée par les noms propres et les descriptions définies pose un problème de taille : quelle est la valeur de vérité d'un énoncé comportant un nom propre ou une description définie sans dénotation?

Avant de présenter les réponses apportées à cette question par les trois auteurs cités, il convient de rappeler que ceux-ci s'inscrivent dans deux courants opposés de la philosophie analytique. Philosophes du langage «idéal », Frege et Russell considèrent que les problèmes philosophiques sont engendrés par les imperfections du langage ordinaire ; pour les résoudre, il est nécessaire de construire un langage artificiel parfait. Strawson fait quant à lui partie des philosophes du langage « ordinaire » pour qui c'est à travers l'analyse du langage ordinaire que les problèmes philosophiques peuvent être résolus.

\subsection{La philosophie du langage idéal}

Frege (1892) est le premier à avoir recours de façon explicite à la notion de présupposition. L'exemple qu'il étudie est devenu célèbre, il s'agit de l'énoncé (7) : Kepler est mort dans la misère. L'analyse proposée par Frege peut être résumée comme suit : Contrairement à ce que l'on pourrait croire, la pensée $<$ Le nom Kepler désigne un individu $>$ n'est pas contenue dans le sens de (7). Si tel était le cas, cette pensée disparaîtrait sous la négation. Or force est de constater qu'elle «vaut tout autant» pour (7) que pour (8) Kepler n'est pas mort dans la misère. On considérera donc que cette pensée est «présupposée ».

Que se passe-t-il si l'individu dont l'existence est présupposée n'existe pas ? Pour Frege, l'énoncé (7) n'est dans ce cas ni vrai ni faux : il n'a pas de référence. On remarquera que, dans le cadre de la logique classique, cette approche soulève un important problème. Depuis Aristote, il est admis qu'un énoncé est soit vrai, soit faux. Il n'y a pas de troisième possibilité. Ce principe est connu sous le nom de «principe de bivalence » ou «principe du tiers exclu». Comme nous venons de le voir, la proposition de Frege remet en cause ce principe.

En proposant d'analyser l'énoncé (9), L'actuel roi de France est chauve, comme une conjonction de propositions, Russell (1905) prend le contrepied de l'approche de Frege (1892). Dans la «théorie des descriptions » qu'il défend, (9) véhicule deux propositions : (9a) < Il existe un homme et un seul qui a la propriété d'être roi de France actuellement > et (9b) < cet homme a la propriété d'être chauve >. Pour Russell, la proposition (9a) est donc un composant du sens de (9) au même titre que la proposition (9b). Si elle est fausse, l'énoncé (9) est faux. Contrairement à la proposition de Frege, cette approche est compatible avec le principe du tiers exclu.

Qu'en est-il de l'énoncé (10), L'actuel roi de France n'est pas chauve? Russell considère que cet énoncé peut être interprété de deux façons différentes, selon que la négation porte sur le quantificateur < il existe > ou sur le prédicat < être chauve >. Dans le premier cas, (10) équivaut à (10a) < Il est faux qu'il y ait une entité qui est actuellement roi de France et qui est chauve > ; dans le second, (10) équivaut à $(10 \mathrm{~b})<\mathrm{Il}$ y a une entité qui est actuellement roi de France et qui n'est pas chauve $>$. 
Version pre-print. A paraître dans Langages.

\subsection{La philosophie du langage ordinaire}

L'un des principaux arguments avancés par Strawson (1950) contre l'approche de Russell (1905) concerne le concept de fausseté. Selon lui, dire que l'énoncé (11) Le roi de France est sage (l'exemple a un peu changé en cinquante ans!) est faux n'équivaut jamais à nier l'existence du roi de France. Si l'existence du roi de France est en un certain sens «impliquée » par l'énoncé (11), il ne s'agit cependant pas d'une implication logique. En énonçant (11), le locuteur n'asserte pas qu'il existe un roi de France mais «signale » qu'il réfère à un individu en l'existence duquel il croit. Si cet individu n'existe pas, la question de savoir si (11) est vrai ou faux «ne se pose pas » car l'acte de référence a échoué.

L'approche de Strawson a été très influente, aussi bien en logique qu'en linguistique. Dans la mesure où elle remet en cause le principe du tiers exclu, cette approche n'est pas compatible avec la logique bivalente classique. Pour résoudre ce problème, certains logiciens ont construit des systèmes à deux valeurs autorisant les «trous », c'est-à-dire les phrases sans valeur de vérité. D'autres ont proposé des systèmes à trois valeurs. En linguistique, l'approche de Strawson a donné lieu à ce que de nombreux manuels appellent la « définition sémantique de la présupposition ». Selon cette définition, le présupposé est une condition nécessaire pour qu'une phrase déclarative ait une valeur de vérité.

\subsection{Bilan}

On aura remarqué que, bien qu'elles s'inscrivent dans des perspectives différentes, les approches de Frege (1892) et de Strawson (1950) présentent de grandes similitudes. Au moins trois points de divergence opposent ces auteurs à Russell (1905). Le premier concerne l'observation des énoncés négatifs : alors que pour Russell l'énoncé (10) peut recevoir deux interprétations différentes (10a et 10b), Frege et Strawson n'interprètent cet énoncé que d'une seule façon (10b). Par ailleurs, il n'y a pas de consensus sur les conséquences logiques de l'échec référentiel : s'il n'existe pas de roi de France, (9) est faux pour Russell et ni vrai ni faux pour Frege et Strawson. Enfin, contrairement à Frege et Strawson, Russell n'accorde aucun statut particulier au présupposé.

Qui a raison? Du point de vue logique, il semble difficile de trancher. Ainsi que le montre Kleiber (1981 : 188-194), les deux approches (celle de Russell d'une part et celle de Frege et Strawson d'autre part) reposent sur des postulats de départ différents (dans un cas, on fait l'hypothèse qu'un énoncé est soit vrai, soit faux ; dans l'autre on introduit la possibilité qu'un énoncé soit inévaluable), des concepts de fausseté différents (dans un cas tous les énoncés présentant un échec référentiel ou prédicatif sont considérés comme faux ; dans l'autre seul l'échec prédicatif entraîne la fausseté) et des questionnements différents (dans un cas, l'objectif est de transcrire les énoncés du langage naturel en langage logique; dans l'autre c'est la description du langage naturel qui prévaut).

Du point de vue linguistique, en revanche, l'inadéquation de l'approche de Russell (1905) est patente. En considérant que (10) peut indiquer qu'il n'y a pas de roi de France, Russell creuse un fossé entre logique et langage ordinaire. Isolée, cette phrase ne peut en aucun cas être interprétée de cette façon; pour qu'elle ait cette interprétation, il faut nécessairement la compléter: (12) Le roi de France n'est pas chauve puisqu'il n'y a pas de roi de France. Par ailleurs, en mettant sur le même plan l'existence du roi de France et sa calvitie, Russell ne 
Version pre-print. A paraître dans Langages.

rend pas compte du fait que ces éléments ne sont pas présentés de la même façon par l'énoncé. Or cette différence est un observable que tout modèle théorique doit prendre en considération (cf. Ducrot, 1972 : 32).

\section{Actes de langage, croyances d'arrière-plan, conditions de félicité : les approches pragmatiques de la présupposition}

C'est à la fin des années 60 que les linguistes commencent à s'intéresser à la présupposition. Très vite, une littérature abondante paraît sur la question: les exemples de constructions présuppositionnelles se multiplient, tout comme les théories destinées à en rendre compte. Ce foisonnement coïncide - et ce n'est pas un hasard - avec le développement de la pragmatique comme sous-discipline de la linguistique : pour la plupart des auteurs de cette époque, le phénomène présuppositionnel doit être décrit en termes d'attitudes et de croyances des participants à la conversation.

Si cette problématique est commune aux différents auteurs que nous réunissons dans cette section, un point de divergence fondamental est cependant à signaler, qui concerne la conception des rapports entre sémantique et pragmatique. Pour Fillmore (1970) et Stalnaker (1974), le composant pragmatique est indépendant du composant sémantique : la prise en compte des attitudes et des croyances des participants à la conversation vient se surajouter à une description sémantique indépendante de ce type de considération. Pour Ducrot (1972, 1980) et Anscombre (1990b), en revanche, la pragmatique est «intégrée » à la sémantique : il n'existe pas de niveau fondamental de signification indépendant de toute pragmatique (cf. Ducrot, 1995 : 131-135).

\subsection{La présupposition pragmatique}

Dans l'approche de Fillmore (1970), les aspects présuppositionnels d'une situation de communication verbale renvoient aux « conditions qui doivent être satisfaites pour qu'un acte illocutionnaire précis soit effectivement accompli en prononçant certaines phrases » (Fillmore, 1970 : 60). Par exemple, l'énoncé (13) Fermez la porte s'il vous plait présuppose (13a) que l'interlocuteur comprend le français, (13b) qu'il ne se trouve pas hors de portée de voix au moment de l'énonciation, (13c) que la relation entre le locuteur et l'interlocuteur permet à celui-ci d'adresser sa demande à celui-là, (13d) que l'interlocuteur est dans une situation qui lui permet de fermer la porte, (13e) que le locuteur a dans l'esprit une certaine porte et (13f) que la porte en question est ouverte au moment de l'énonciation. Toutes ces conditions peuvent en effet être vues comme des conditions préalables à l'emploi de (13) et il est à noter qu'aucune d'entre elles n'est modifiée par la négation. Cependant, toutes n'intéressent pas le linguiste : contrairement aux autres conditions, (13a) et (13b) ne sont pas liées à la structure linguistique de (13) et ne jouent pas de rôle particulier dans la compréhension de cette phrase; pour Fillmore, elles peuvent donc être ignorées par le linguiste.

Si l'approche de Fillmore (1970) est peu citée à l'heure actuelle, l'article de Stalnaker (1974) est en revanche considéré comme fondateur par de nombreux auteurs (cf. par exemple Geurts, 1999 et Simons 2001, 2006). Pour Stalnaker, les présupposés sont des croyances d'arrièreplan, c'est-à-dire des propositions que le locuteur tient pour vraies ou semble tenir pour vraies au moment de l'énonciation. Dire qu'un locuteur présuppose P (pour Stalnaker, ce ne sont pas 
les phrases qui présupposent mais les locuteurs) équivaut à dire que le locuteur est disposé à agir comme si $\mathrm{P}$ faisait partie de l'ensemble des informations d'arrière-plan qu'il partage avec son interlocuteur dans le cadre de la conversation qui est la leur. Les présupposés sont ainsi vus comme des contraintes sur ce qui peut être dit de façon appropriée dans le contexte qu'ils définissent. Par exemple, il n'est approprié d'énoncer (14) La reine d'Angleterre est chauve que dans un contexte où le fait que l'Angleterre a une reine fait partie des connaissances d'arrière-plan présumées.

\subsection{La pragmatique intégrée}

Ducrot a proposé, à dix ans d'intervalle, deux approches très différentes de la présupposition. La première (Ducrot, 1972) s'inspire de la théorie d'Austin sur les actes de langage. Pour Ducrot, les actes de langage peuvent être considérés comme des actes juridiques accomplis par la parole. Créant des droits et des devoirs, ils transforment la situation juridique des participants à la conversation. Par exemple, poser une question, c'est créer une obligation de réponse. Si l'interlocuteur ne répond pas, il commet un acte d'impolitesse. De même, choisir un énoncé comportant tel ou tel présupposé, c'est obliger l'interlocuteur à prendre ce contenu pour cadre de sa propre parole dans la suite du dialogue. Refuser les présupposés d'un énoncé équivaudrait en effet à disqualifier l'acte de parole accompli par le locuteur et serait perçu comme une attaque.

Ducrot (1980) propose une autre caractérisation de la présupposition, s'inscrivant cette fois dans le cadre de la théorie de la polyphonie. Selon cette théorie, un locuteur ne se présente pas toujours comme source de ce qu'il dit. Un même énoncé peut mettre en scène différents points de vue, attribués à différentes sources. Dans ce cadre, la présupposition renvoie à l'opinion commune, à laquelle se rallie le locuteur :

Admettons [...] que la présupposition soit elle-même [...] un fait de polyphonie. En disant Pierre continue à fumer, on présenterait le fait que Pierre ait fumé autrefois comme asserté par un premier énonciateur, distinct du locuteur et souvent identifié avec l'opinion commune (énonciateur sur lequel le locuteur fonde sa propre croyance); dans cette hypothèse, le locuteur ne prend personnellement à son compte que la deuxième affirmation, celle de Pierre fume actuellement, autrement dit, il est identifié avec le seul énonciateur de cette affirmation, c'est-à-dire, selon ma terminologie, du posé. (Ducrot, $1980: 51$ )

Malgré leurs différences, les deux approches présentées s'inscrivent dans une même perspective théorique. Dans ses différents travaux, Ducrot insiste sur le caractère proprement linguistique du phénomène présuppositionnel. Les présupposés constituent selon lui un « implicite immédiat » (par opposition à un « implicite discursif » lié à l'acte d'énonciation) et doivent être conçus non comme des conditions d'emploi mais comme des éléments de contenu. La possibilité de présenter certains contenus comme acquis et indiscutables et d'obliger un interlocuteur à adopter un certain cadre de discours est en fait inscrite dans la langue même, indépendamment de son utilisation. Pour Ducrot, cette conception confirme l'idée que la langue est « vouée à l'interaction des individus » et que « la pragmatique n'est pas un supplément à la sémantique »(Ducrot, $1984: 44)$.

Anscombre (1990b) remarque que thème, présupposé et assertion préalable sont trois manifestations particulières d'un même phénomène : il s'agit « d'espaces discursifs dominants » dans lesquels viennent s'emboîter des «espaces discursifs dominés ». Par 
Version pre-print. A paraître dans Langages.

exemple, dans les trois énoncés suivants, le contenu (a) est présenté comme le cadre à l'intérieur duquel doit être envisagé le contenu (b) :

(15) Hier Pierre est venu.

$15 \mathrm{a}-<\mathrm{Il}$ s'est passé des choses hier $>$ (thème) / 15b- < Pierre est venu >

(16) Pierre a cessé de fumer.

16a- < Pierre fumait avant > (présupposé) / 16b- < Pierre ne fume pas actuellement $>$

(17) Si Pierre est gentil, je l'emmène au cinéma.

17a- < Pierre est gentil > (assertion préalable) / 17b- < J'emmène Pierre au cinéma >

Dans la continuité des travaux de Ducrot, Anscombre propose de rendre compte de la différence entre les trois types d'espaces discursifs dans un cadre polyphonique, en termes de position du locuteur vis-à-vis de l'un ou de l'autre. Alors que la position du locuteur par rapport à une assertion préalable peut varier (rien en (17) n'indique si le locuteur adhère ou non au contenu 17a), il n'en va pas de même pour le thème et le présupposé : le présupposé est présenté comme le point de vue d'une communauté à laquelle le locuteur dit appartenir (c'est pourquoi le locuteur de (16) ne peut en aucun cas refuser d'adhérer au contenu 16a) tandis que le thème est attribué à une communauté à laquelle le locuteur peut ou non appartenir (en (15) le locuteur peut ou non adhérer au contenu 15a).

\subsection{Bilan}

En adoptant une approche pragmatique de la présupposition, les auteurs réunis dans cette section cherchent avant tout à rendre compte du statut particulier du présupposé. Si l'on excepte la proposition de Fillmore (1970), on peut considérer qu'il y a consensus sur la façon de caractériser ce statut. Pour Stalnaker (1974), Ducrot (1972, 1980) et Anscombre (1990b), le présupposé est présenté par l'énoncé comme un élément d'arrière-plan, appartenant à un «passé de la connaissance » (Ducrot, $1984: 21$ ) commun au locuteur et à son interlocuteur et ne faisant pas l'objet de la communication.

Contre cette conception, Burton-Roberts (1989) met en avant le fait que le présupposé constitue souvent une information nouvelle pour l'interlocuteur : "If I were to say to you, $M y$ sister is coming to lunch tomorrow, I do presuppose that I have a sister but in presupposing it I do not necessarily assume that you have a prior assumption or belief that I have a sister" (Burton-Roberts, 1989 : 26). Cette critique est difficilement recevable : les auteurs cités ne disent pas que le présupposé est une information connue mais qu'il est présenté comme une information connue. Le locuteur fait comme si son interlocuteur connaissait l'information présupposée et il s'attend à ce que son interlocuteur fasse comme si, effectivement, il connaissait cette information. La « règle de compromis » ("rule of accomodation") proposée par Lewis (1979) formalise ce mécanisme basé sur la bonne volonté de l'interlocuteur : "If at time $\mathrm{t}$ something is said that requires presupposition $\mathrm{P}$ to be acceptable, and if $\mathrm{P}$ is not presupposed just before $t$, then [...] presupposition P comes into existence at $t$ " (Lewis, 1979: 340).

\section{Les approches réductionnistes : a Requiem for Presupposition}

Pour les défenseurs de l'approche réductionniste, il n'y a pas lieu de distinguer présupposition et assertion dans la grammaire : les phénomènes qualifiés de «présuppositionnels » dans la 
Version pre-print. A paraître dans Langages.

littérature partagent certaines propriétés avec différents types d'inférences pragmatiques et peuvent être étudiés dans des cadres déjà établis.

A la suite de Simons (2006), nous distinguerons deux types de réductionnisme. Le premier, appelé par Simons «réductionnisme phénoménologique », nie qu'il y ait un quelconque avantage descriptif à avoir recours à la notion de présupposition. Le second, appelé «réductionnisme explicatif » et basé sur l'hypothèse de la sous-détermination linguistique, cherche à rendre compte des propriétés des présupposés en faisant appel à des principes généraux tels que les maximes de Grice ou le principe de pertinence de Sperber et Wilson.

\subsection{Le réductionnisme phénoménologique}

Dans un article intitulé "Requiem for Presupposition", Karttunen \& Peters (1977) mettent en question le concept de présupposition en affirmant que les phénomènes regroupés sous cette étiquette sont très hétérogènes et ne peuvent faire l'objet d'un traitement unifié. Après avoir énuméré les différentes constructions qualifiées de « présuppositionnelles » par les linguistes, Karttunen \& Peters centrent leur étude sur plusieurs cas particuliers et montrent, pour chacun, que le recours à la notion de présupposition est non seulement erroné mais aussi inutile car les phénomènes ainsi décrits peuvent être expliqués au moyen de catégories générales telles que les implicatures conversationnelles (particulières et généralisées), les implicatures conventionnelles ou encore les conditions de félicité.

Même si les deux auteurs semblent se refuser à conclure de façon radicale sur un possible abandon de la notion de présupposition, il s'agit là d'un exemple de réductionnisme phénoménologique puisque c'est l'utilité même de la notion qui est discutée dans cet article.

\subsection{Le réductionnisme explicatif}

Alors que l'approche traditionnelle fait de la présupposition une relation nécessairement invariante, les défenseurs du réductionnisme explicatif multiplient les exemples d'annulation des présupposés. Ce faisant, ils mettent en question le statut spécifique du présupposé en le rapprochant de celui de l'implicature conversationnelle :

\section{Annulabilité sous la négation :}

(18) Le roi de France n'est pas chauve puisqu'il n'y a pas de roi de France (Wilson, 1975) : Le présupposé < Il y a un roi de France > habituellement véhiculé par Le roi de France n'est pas chauve n'est pas conservé en (18).

\section{Annulabilité dans les phrases complexes :}

(19) Ou la reine de Tonga gouverne despotiquement, ou il n'y a pas de reine de Tonga (Wilson, 1975) : Le présupposé < Il y a une reine de Tonga > habituellement véhiculé par La reine de Tonga gouverne despotiquement n'est pas conservé en (19).

Annulabilité dans certains contextes spécifiques :

(20) Je ne sais pas que Paul est venu (Levinson, 1983 - repris par Moeschler, 1994) : Alors que la même phrase à la troisième personne (par exemple Jean ne sait pas que Paul est venu) présupposerait que < Paul est venu >, (20) ne véhicule pas cette présupposition.

\section{Annulabilité dans certaines situations de discours :}

(21) Au moins Pierre ne regrettera pas d'avoir soutenu sa thèse (Levinson, 1983 - repris par Moeschler, 1994) : Cet énoncé peut être utilisé dans une situation de discours où le locuteur et son interlocuteur savent que Pierre ne soutiendra pas sa thèse. Dans une telle situation, le présupposé < Pierre soutiendra sa thèse > habituellement véhiculé par Pierre ne regrettera pas d'avoir soutenu sa thèse est annulé. 
Le réductionnisme explicatif a été défendu par de nombreux auteurs (Atlas, 1977 ; Kempson, 1975 ; Wilson, 1975 ; Sperber \& Wilson, 1979 ; Atlas \& Levinson, 1981 ; Grice, 1981 ; Simons, 2001), sous des formes et dans des cadres très divers. Mais, si les hypothèses explicatives varient d'un article à un autre, toutes s'appuient sur les mêmes principes de base, qui peuvent être résumés en trois points (cf. Kleiber, $1981: 201$ et Simons, $2006: 363$ ) : (i) les «présupposés » des phrases affirmatives sont en fait des implications, (ii) c'est pour des raisons conversationnelles que ces implications apparaissent en arrière-plan et (iii) c'est pour des raisons conversationnelles que ces implications semblent se conserver sous la négation et l'interrogation.

Pour illustrer la démarche réductionniste, nous prendrons l'exemple de l'article de Sperber \& Wilson (1979). Pour ces auteurs, toutes les implications d'une phrase ne sont pas du même niveau : il y a des implications de premier-plan et des implications d'arrière-plan. Les implications d'arrière-plan d'une phrase sont obtenues en remplaçant les constituants de la phrase par des variables. Par exemple, l'énoncé (22) Peter is married to SARAH véhicule les implications (22a) < Peter est marié à Sarah >, (22b) < Peter est marié à quelqu'un >, (22c) < Peter a une propriété > et $(22 d)<$ Quelque chose est le cas >. (22a) est une implication de premier-plan tandis que (22b), (22c) et (22d) sont des implications d'arrière-plan. Quant au présupposé, il correspond à la première implication d'arrière-plan, ici (22b). Lors de l'énonciation, les implications d'arrière-plan sont considérées comme non pertinentes car l'énonciation concerne seulement les implications de premier-plan. C'est pourquoi, sous la négation et l'interrogation, seules les implications de premier-plan sont niées ou mises en question. Ainsi, il est possible d'expliquer les propriétés des présupposés en ayant recours uniquement à des principes conversationnels et sans accorder de statut spécifique à la présupposition dans la grammaire.

\subsection{Bilan}

Comme nous l'avons vu, l'argument principal qu'avancent les défenseurs du réductionnisme explicatif pour justifier le traitement conversationnel des présupposés est leur annulabilité. Cette approche a soulevé de nombreuses objections dans la littérature. Le premier type d'objection concerne l'acceptabilité des exemples d'annulation proposés par les auteurs. La réaction de Kleiber (1981) face aux exemples présentés par Wilson (1975) est significative à cet égard: "A l'examen, les cinq séries d'énoncés proposés par D. Wilson ne se révèlent guère probants, certains d'entre eux étant anormaux, d'autres interprétés incorrectement » (Kleiber, 1981 : 204). Ce commentaire pourrait également s'appliquer à l'exemple (20). Selon nous, cet énoncé n'est recevable que dans un contexte très particulier où le locuteur, qui sait que Paul est venu, s'engage, parce qu'on le lui demande, à faire comme s'il ne le savait pas. En dehors de ce type de contexte, (20) ne peut qu'être rejeté. Le fait que certains auteurs en proposent une version attestée ${ }^{2}$ ne remet pas en cause notre objection : les observables, ce ne sont pas les exemples attestés mais les jugements d'acceptabilité du linguiste.

Le concept d'annulabilité a lui aussi fait l'objet de critiques. Distinguant trois types d'annulation (l'annulation proprement dite, la «méta-annulation» et la «suspension»), Amsili (2007) montre que le comportement des présupposés ne peut en aucun cas être

\footnotetext{
${ }^{2}$ C'est le cas par exemple de Beaver \& Geurts (2011), qui citent une intervention du général Dan McNeill : I don't know that Mullah Omar is alive. I don't know if he's dead either (Reuters, 19/05/2008).
} 
Version pre-print. A paraître dans Langages.

assimilé à celui des implicatures conversationnelles. Non seulement les présupposés ne sont pas annulables au sens propre (* Le roi de France est chauve, mais il n'y a pas de roi de France) mais en plus, force est de constater que la «méta-annulation » n'est possible que dans le cas des présupposés d'existence (Le roi de France n'est pas chauve puisqu'il n'y a pas de roi de France) ; avec les autres types de constructions présuppositionnelles elle donne lieu à des enchaînements inacceptables (*Ce n'est pas à Tombouctou qu'il est allé, il n'a pas bougé de chez lui!). Pour Amsili, il n'est finalement possible de parler d'annulation que dans les cas de «suspension » (Ou la reine de Tonga gouverne despotiquement, ou il n'y a pas de reine de Tonga). Nous reviendrons sur ce point dans la dernière section de cet article, lorsque nous aborderons le problème de la projection.

Nous avons vu que, pour les défenseurs du réductionnisme, la conservation des présupposés sous la négation était un phénomène conversationnel. Cette conception implique que l'énoncé (23) Le roi de France n'est pas chauve a pour interprétation première (23a) < Il est faux qu'il $\mathrm{y}$ ait une entité qui est actuellement roi de France et qui est chauve $>$ et que l'interprétation (23b) < Il y a une entité qui est actuellement roi de France et qui n'est pas chauve > est dérivée conversationnellement. Kleiber (1981) prend le contrepied de cette approche en démontrant qu'elle est difficilement compatible avec les principes gricéens :

Admettons que je veuille asserter qu'il n'existe pas de roi de France. Il est évident que je n'utiliserai pas l'énoncé (21) $[=(23)]$. Deux raisons expliquent cette décision :

1- (21) [= (23)] ne représente pas le chemin le plus court pour véhiculer cette information. Un locuteur qui énonce (21) [= (23)] pour exprimer (21b) [= (23a)] contrevient à la loi gricéenne de la brièveté.

2- D'un autre côté, étant donné qu'il existe un moyen plus simple d'exprimer (21b) [= (23a)], un locuteur risque en énonçant un énoncé plus complexe d'induire en erreur son interlocuteur. Il s'agit d'une autre loi conversationnelle de Grice, «the avoidance of obscurity or ambiguity ». Seule l'interprétation (21a) [= (23b)] reste donc possible (Kleiber, $1981: 209)$.

Rappelons que, pour une majorité de linguistes, le problème posé par (23) ne concerne pas l'interprétation (23b) - qui correspond à la lecture naturelle de la négation - mais au contraire (23a) : comment expliquer que la négation ne conserve pas toujours les présupposés? Pour répondre à cette question, plusieurs auteurs ont proposé de distinguer deux types de négation : la négation descriptive, qui conserve les présupposés, et la négation métalinguistique, qui les réfute (cf. Ducrot, 1972 ; Horn, 1985). Ils parviennent ainsi à expliquer l'acceptabilité d'enchaînements tels que (18) sans remettre en cause l'hypothèse de l'invariance de la relation de présupposition.

\section{La problème de la projection et le tournant dynamique}

Les approches que nous réunissons dans cette dernière section se présentent comme autant de réponses à un problème que nous n'avons pas encore abordé : le problème de la «projection » des présupposés. Ce problème peut être formulé de la façon suivante: comment les présupposés d'une phrase complexe sont-ils déterminés par les présupposés des propositions qu'elle comporte ? Avec les « donkey sentences », cette question est à l'origine du « tournant dynamique » en sémantique formelle (cf. Schlenker, 2008) : la phrase n'est plus conçue comme «capturant la classe des modèles où elle est (statiquement) vraie » mais est désormais envisagée comme «modifiant (dynamiquement) le contexte de l'interprétation du discours ultérieur » (Rebuschi et Tulenheimo, $2004: 2$ ). 
Version pre-print. A paraître dans Langages.

Après avoir exposé les premières approches du problème de la projection (Karttunen, 1973b ; Gazdar, 1979), nous présenterons deux modèles dynamiques s'inscrivant dans deux cadres différents : la théorie de la satisfaction de Heim (1983), qui relève de la CCS ("ContextChange Semantics") et l'approche anaphorique de van der Sandt (1992), qui relève de la DRT ("Discourse Representation Theory").

\subsection{Le problème de la projection : premières approches}

Avant d'en sonner le glas en 1977, Karttunen a beaucoup travaillé sur la présupposition ${ }^{3}$. C'est par exemple lui qui, en 1973, pose le problème de la projection des présupposés. S'opposant à «l'hypothèse cumulative » de Langendoen \& Savin (1971) selon laquelle la phrase complexe hérite les présupposés des propositions qu'elle comporte, Karttunen propose une typologie des prédicats en fonction de leur comportement vis-à-vis des présupposés. Il distingue ainsi trois catégories différentes :

1- Les « bouchons » (“plugs") sont des prédicats qui bloquent toujours la projection des présupposés. Appartiennent à cette catégorie les verbes de dire tels que promettre, prévenir, accuser, etc.

Par exemple, l'énoncé (24) Harry a promis à Bill de le présenter au roi de France actuel ne présuppose pas que $<$ le roi de France existe $>$.

2- Les « trous » (“holes”) sont des prédicats qui ne bloquent jamais la projection des présupposés. Appartiennent à cette catégorie des verbes tels que savoir, surprendre, hésiter, cesser, etc. Par exemple, l'énoncé (25) Cécilia savait que cela surprenait Marie que Fred hésite à cesser de battre sa femme présuppose que < Fred battait sa femme auparavant >, tout comme la phrase simple Fred a cessé de battre sa femme.

3- Les « filtres » (“filters") sont des prédicats qui bloquent la projection des présupposés sous certaines conditions.

Appartiennent à cette catégorie les opérateurs de condition ( $s i$... alors), de conjonction (et) et de disjonction (soit... soit).

Par exemple, le présupposé < Jack a des enfants > véhiculé par la phrase simple Tous les enfants de Jack sont chauves apparaît en (26) Si la calvitie est héréditaire, alors tous les enfants de Jack sont chauves et en (27) Soit la calvitie n'est pas héréditaire, soit tous les enfants de Jack sont chauves mais pas en (28) Si Jack a des enfants, alors tous les enfants de Jack sont chauves ni en (29) Soit Jack n'a pas d'enfants, soit tous les enfants de Jack sont chauves.

En plus de cette typologie, Karttunen énonce des règles rendant compte du comportement de chaque «filtre». Dans le cas de l'opérateur conditionnel par exemple, la règle est que le présupposé de l'apodose ne devient un présupposé de la phrase complexe que s'il n'est pas impliqué par la protase.

Une autre solution au problème de la projection est proposée par exemple par Gazdar (1979). Dans son modèle, l'interlocuteur doit, pour interpréter un énoncé, ajouter les différentes propositions véhiculées par cet énoncé à l'ensemble des propositions connues et acceptées par les participants à la conversation (le « contexte »). Ce mécanisme d'ajout s'applique d'abord aux implications de l'énoncé, puis aux implicatures conversationnelles et enfin aux présupposés. A chaque étape, les propositions additionnelles ne peuvent être prises en compte que si elles sont compatibles avec les propositions déjà présentes dans le contexte. Par

\footnotetext{
${ }^{3}$ Mais il n'est pas étonnant qu'il ait fini par « enterrer» la notion. Comme le souligne Geurts (1999: 5) : « An especially stark illustration of the disparity of the field, at least in its early days, is the work of a Karttunen, who within the span of six years published three theories that were mutually inconsistent, technically as well as conceptually » (i.e. Karttunen 1973, 1974, and Karttunen and Peters 1979). »
} 
Version pre-print. A paraître dans Langages.

exemple, en (28), la protase (Si Jack a des enfants) indique que le locuteur ne sait pas si Jack a des enfants ou non (selon Gazdar il s'agit d'une implicature conversationnelle). Cette implicature entrant en conflit avec le présupposé véhiculé par l'apodose (Tous les enfants de Jack sont chauves présuppose que < Jack a des enfants >), le présupposé est annulé.

\subsection{Les modèles dynamiques}

S'inspirant des travaux de Stalnaker, Heim (1983) propose de traiter la présupposition dans un cadre dynamique, en termes de changement de contexte. Selon elle, l'introduction d'une phrase $\mathrm{P}$ dans un contexte $\mathrm{c}$ a pour fonction de «mettre à jour » $\mathrm{c}$, c'est-à-dire d'ajouter le contenu de $\mathrm{P}$ à $\mathrm{c}$. Dans ce cadre, les présupposés activés par une phrase sont vus comme des contraintes sur l'ensemble des contextes susceptibles d'être mis à jour par cette phrase. Dire que (30) Le roi a un fils présuppose qu'il y a un roi équivaut à dire que (30) ne peut être admise que dans des contextes impliquant (30a) $<$ Il y a un roi $>$.

Pour traiter le problème de la projection, Heim s'appuie sur Karttunen (1974) et postule que, dans le cas des phrases complexes, le contexte est mis à jour «localement» par chaque constituant de la phrase. Elle propose ainsi de rendre compte de la projection des présupposés dans les conditionnelles sur la base des trois règles suivantes :

(10) If "If A, B" is uttered in a context $\mathrm{c}$, then $\mathrm{c}$ is the local context for A, and c + A (read : "c incremented by A") is the local context for B.

(11) A context $\mathrm{c}$ admits a sentence $\mathrm{S}$ just in case each of the constituent sentences of $\mathrm{S}$ is admitted by the corresponding local context [...]

(12) $\mathrm{S}$ presupposes $\mathrm{p}$ iff all contexts that admit $\mathrm{S}$ entail $\mathrm{p}$ (Heim, 1983: 116).

Appliquons ces règles à la phrase (31) Si le roi a un fils alors le fils du roi est chauve. (31) est constituée de deux phrases : (30) Le roi a un fils (qui présuppose, nous l'avons vu, (30a) $<$ Il y a un roi >) et (32) Le fils du roi est chauve (qui présuppose (30a) < Il y a un roi > et (32a) < Le roi a un fils >). Enoncée dans un contexte c, (31) fait intervenir deux contextes locaux : c est le contexte local pour (30) tandis que $\mathrm{c}+(30)$ est le contexte local pour (32). Par ailleurs, étant donné le contenu présuppositionnel de (30) et (32), (i) (30) ne peut être admise dans c que si c implique (30a) et (ii) (32) ne peut être admise dans c + (30) que si c + (30) implique (30a) et (32a). On remarquera que si la condition (i) est satisfaite alors la condition (ii) l'est également puisque (32a) est impliquée par (30). Par conséquent, la seule condition pour que (31) soit admise dans c est que c implique (30a). Autrement dit, le seul présupposé activé par (31) dans sa globalité est (30a). Les trois règles proposées par Heim permettent ainsi d'expliquer pourquoi le présupposé (32a) véhiculé par (32) n'apparaît pas en (31) ${ }^{4}$.

Van der Sandt (1992) remarque quant à lui que, sur de nombreux points, les présupposés se comportent comme les anaphores. Sur la base de cette observation, il propose de considérer que, comme les anaphores, les présupposés doivent être « résolus ", c'est-à-dire qu'il faut leur trouver un antécédent. Cette résolution peut se faire soit par liage (comme dans le cas des pronoms, l'antécédent est recherché dans le discours lui-même), soit par accomodation (contrairement à ce qui se passe dans le cas des pronoms, le contenu descriptif des constructions présuppositionnelles leur permet de se créer un antécédent si le discours n'en

\footnotetext{
${ }^{4}$ Il s'agit là de la première version du modèle de Heim (1983), avant l'introduction du concept de « potentiel de changement de contexte » ("context change potential"). Dans la mesure où cette version est suffisante pour comprendre la démarche de cet auteur (et surtout, faute de place), nous n'entrons pas dans les détails.
} 
Version pre-print. A paraître dans Langages.

fournit pas). Dans le cadre de la DRT, van der Sandt fournit un ensemble de règles permettant de rendre compte de la résolution des présupposés selon ces deux modes, ainsi que du problème de la projection.

\subsection{Bilan}

Pour conclure cette section, nous voudrions nous interroger sur la formulation même du problème de la projection. Dans les différentes études citées, le comportement des «filtres » est uniquement vu comme un problème lié à la présupposition : personne ne cherche à caractériser les différents opérateurs regroupés sous cette étiquette. Par exemple, après avoir montré que les présupposés disparaissaient dans certains enchaînements disjonctifs, Beaver (2001) écarte d'un revers de main (dans une note de bas de page) la question du sens de l'opérateur de disjonction :

One aspect of these examples which I will not consider in detail is the presence of the word either. As Prince $(1978$, p.372) pointed out, the presence of this word is essential to the felicity of many examples where a presupposition triggered in a disjunction is cancelled. Prince conjectures that the either acts as a signal to the hearer to «delay attribution 》 of information in the disjuncts, which she suggests may lead to the presuppositions not being regarded as beliefs of the speaker. (Beaver, 2001 : note 14 p. 44)

En fait, il semble que les auteurs cités assimilent systématiquement le sens des différents «filtres » au sens des opérateurs logiques correspondants. Or il est possible de considérer que la différence entre les phrases où les présupposés sont conservés et les phrases où les présupposés disparaissent est due, au moins en partie, au fonctionnement de ces opérateurs. Par exemple, on remarque que (26) et (28) illustrent deux emplois très différents de l'opérateur conditionnel : alors que (26) introduit une relation de cause à conséquence entre la protase et l'apodose, il n'en va pas de même en (28). Par ailleurs, seul (26) admet la commutation (en 26a) et la contraposition (en 26b) :

(26) Si la calvitie est héréditaire alors tous les enfants de Jack sont chauves.

(26a) Si tous les enfants de Jack sont chauves alors la calvitie est héréditaire.

(26b) Si tous les enfants de Jack ne sont pas chauves alors la calvitie n'est pas héréditaire.

(28) Si Jack a des enfants alors tous les enfants de Jack sont chauves.

(28a) * Si tous les enfants de Jack sont chauves alors Jack a des enfants.

(28b) * Si tous les enfants de Jack ne sont pas chauves alors Jack n'a pas d'enfants.

Peut-on rendre compte de ces différences sans s'interroger sur le fonctionnement de si?

\section{Conclusion}

Tout au long de cet article, nous avons cherché à mettre en évidence l'interdépendance entre les problèmes abordés par les auteurs et le cadre théorique dans lequel ils s'inscrivent. Nous avons ainsi distingué quatre types d'approches, mettant en avant quatre problèmes différents : (i) le problème de l'échec présuppositionnel, au centre de la controverse logicophilosophique, (ii) la question du statut du présupposé, qui fonde l'approche pragmatique, (iii) l'annulabilité des présupposés, argument principal des réductionnistes et enfin (iv) le problème de la projection, à l'origine du «tournant dynamique ». Dans chaque cas, nous avons essayé de montrer que ces problèmes n'étaient pas des problèmes «dans l'absolu ». L'importance qui leur est accordée dépend du cadre théorique dans lequel ils sont envisagés et 
Version pre-print. A paraître dans Langages.

un problème fondamental pour les uns peut être considéré comme secondaire par les autres. Nous conclurons sur ce point en opposant les deux citations suivantes, concernant le problème de l'échec présuppositionnel :

We cannot hope to understand the way someone is using the word presupposition unless we are able to discover what is thought to result when a presupposition fails (Garner, 1971: 27).

The problem of presupposition failure is the oldest one. If a statement presupposes something which isn't true, what are the consequences for the statement? Will it be false or meaningless, or perhaps wasn't it a statement in the first place? These questions have been debated with so much wit and zest that I am almost reluctant to say that I find them of little interest. (Geurts, $1999: 1) .^{5}$

\section{Références bibliographiques}

AmsiLi P. (2007), «L'annulation des implicatures et des présuppositions », Revue de sémantique et pragmatique 21-22, 193-206.

ANSCOMBRE J.C. (1990), «Thème, espaces discursifs et représentation événementielle », in J.C. Anscombre \& G. Zaccaria (éds), Fonctionnalisme et pragmatique, Milan : Unicopli, 43150.

ATLAS J.D. (1977), "Negation, ambiguity, and presupposition”, Linguistics and Philosophy 1, 321-336.

ATLAS J.D. \& LEVINSON S. (1981), "It-clefts, informativeness and logical form: radical pragmatics", in P. Cole (ed.), Radical Pragmatics, New York: Academic Press, 1-61.

BEAVER D. (2001), Presupposition and Assertion in Dynamic Semantics, CSLI Publications.

BEAVER D. \& GEURTS B. (2011), "Presupposition", in Stanford Encyclopedia of Philosophy, < http://plato.stanford.edu/entries/presupposition/index.html >.

BURTON-ROBERTS N. (1989), The limits to debate: A revised theory of semantic presupposition, Cambridge: Cambridge University Press.

DUCROT O. (1972), Dire et ne pas dire, Paris : Hermann.

DUCROT O. (1980), Les mots du discours, Minuit : Paris.

DUCROT O. (1984), Le dire et le dit, Paris : Minuit.

DUCROT O. \& SCHAEFFER J.M. (1995), Nouveau dictionnaire encyclopédique des sciences du langage, Seuil : Paris.

FILLMORE C. (1970), « Verbes de jugement. Essai de description sémantique », Langages 17, $58-72$.

FREGE G. (1892), « Sinn und Bedeutung », traduit par C. Imbert, in Ecrits logiques et philosophiques (Seuil, 1971) sous le titre « Sens et dénotation », 102-126.

GARNER R.T. (1971), "Presupposition in philosophy and linguistics", in C.J. Fillmore \& D.T. Langendoen (eds.), Studies in Linguistic Semantics, New York: Holt, Rinehart \& Winston.

GAZDAR G. (1979), Pragmatics. Implicature, Presupposition and Logical Form, New York: Academic Press.

GEURTS B. (1999), Presupposition and Pronouns, Oxford: Elsevier.

GRICE H.P. (1981), "Presupposition and conversational implicature", in P. Cole (ed.), Radical Pragmatics, New York: Academic Press, 183-198.

HEIM I. (1983), "On the projection problem for presuppositions", WCCFL 2, 114-125.

HORN L. (1985), "Metalinguistic negation and pragmatic ambiguity", Language 61, 121-174.

\footnotetext{
${ }^{5}$ Je remercie J.C. Anscombre et P. Amsili pour leurs commentaires sur des versions antérieures de ce travail.
} 
Version pre-print. A paraître dans Langages.

KARTTUNEN L. (1973), "Presuppositions of compound sentences”, Linguistic Inquiry 4, 169193.

KARTTUNEN L. (1974), "Presupposition and linguistic context”, Theoretical Linguistics 1, 344.

KARTTUNEN L. \& PETERS S. (1977), "Requiem for presupposition”, Proceedings of the Third Annual Meeting of the Bekerley Linguistic Society, 360-371.

KEENAN E.O. (1971), "Two kinds of presuppositions in natural language", in C.J. Fillmore \& D.T. Langendoen (eds.), Studies in Linguistic Semantics, New York: Holt, Rinehart \& Winston.

KEMPSON R.M. (1975), Presupposition and the Delimitation of Semantics, Cambridge: Cambridge University Press.

KLEIBER G. (1981), Problèmes de référence. Descriptions définies et noms propres, Paris : Klincksieck.

LANGENDOEN D.T. \& SAVIN H.B. (1971), "The projection problem for presupposition", in D.D. Steinberg \& L.A. Jakobovits (eds), Semantics. An interdisciplinary Reader in Philosophy, Linguistics, and Psychology, Cambridge: Cambridge University Press.

LEVINSON S.C. (1983), Pragmatics, Cambridge: Cambridge University Press, 167-225.

LEWIS D. (1979), "Scorekeeping in a language game", Journal of Philosophical Logic 8, 339359.

MOESCHLER J. \& REBOUl A. (1994), Dictionnaire encyclopédique de pragmatique, Paris : Seuil.

Rebuschi M. \& TUlenheimo T. (2004), « Des jeux en logique », Philosophia Scientia 8 (2), $1-14$.

RUSSELL B. (1905), “On denoting”, traduit par J.M. Roy, in Ecrits de logique philosophique (PUF, 1989) sous le titre « De la dénotation », 203-218.

SCHLENKER P. (2008), "Be Articulate : A pragmatic theory of presupposition projection", Theoretical Linguistics 34-3, 157-212.

SIMONS M. (2001), "On the conversational basis of some presuppositions", Semantics and Linguistic Theory (SALT) 11, 2001.

SIMONS M. (2006), "Foundational issues in presupposition", Philosophy Compass 1(4), 357372.

SPERBER D. \& WILSON D. (1979), "Ordered entailments: an alternative to presuppositional theories", in C.K. Oh et D.A. Dineen (eds), Syntax and Semantics. Presupposition, New York : Academic Press, 299-323.

STALNAKER R. (1974), "Pragmatic presuppositions", in M. Munitz \& P. Unger (eds), Semantics and philosophy, New York: New York University Press, 197-214.

STRAWSON P.F. (1950), "On referring", traduit par J. Milner, in Etudes de logique et de linguistique (Seuil, 1977) sous le titre « De l'acte de référence », 9-38.

VAN DER SANDT R. (1992), "Presupposition Projection as Anaphora Resolution", Journal of Semantics 9, 333-378.

WILSON D. (1975), Presuppositions and Non-Truth-Conditional Semantics, London: Academic Press. 